This item was submitted to Loughborough's Research Repository by the author.

Items in Figshare are protected by copyright, with all rights reserved, unless otherwise indicated.

\title{
Distributed quasi-orthogonal space-time coding for two-way wireless relay networks
}

PLEASE CITE THE PUBLISHED VERSION

http://dx.doi.org/10.1109/ISWCS.2010.5624515

PUBLISHER

(C) IEEE

VERSION

VoR (Version of Record)

LICENCE

CC BY-NC-ND 4.0

REPOSITORY RECORD

Abdurahman, F.M., A.M. Elazreg, and Jonathon Chambers. 2019. "Distributed Quasi-orthogonal Space-time Coding for Two-way Wireless Relay Networks". figshare. https://hdl.handle.net/2134/7736. 
This item was submitted to Loughborough's Institutional Repository (https://dspace.lboro.ac.uk/) by the author and is made available under the following Creative Commons Licence conditions.

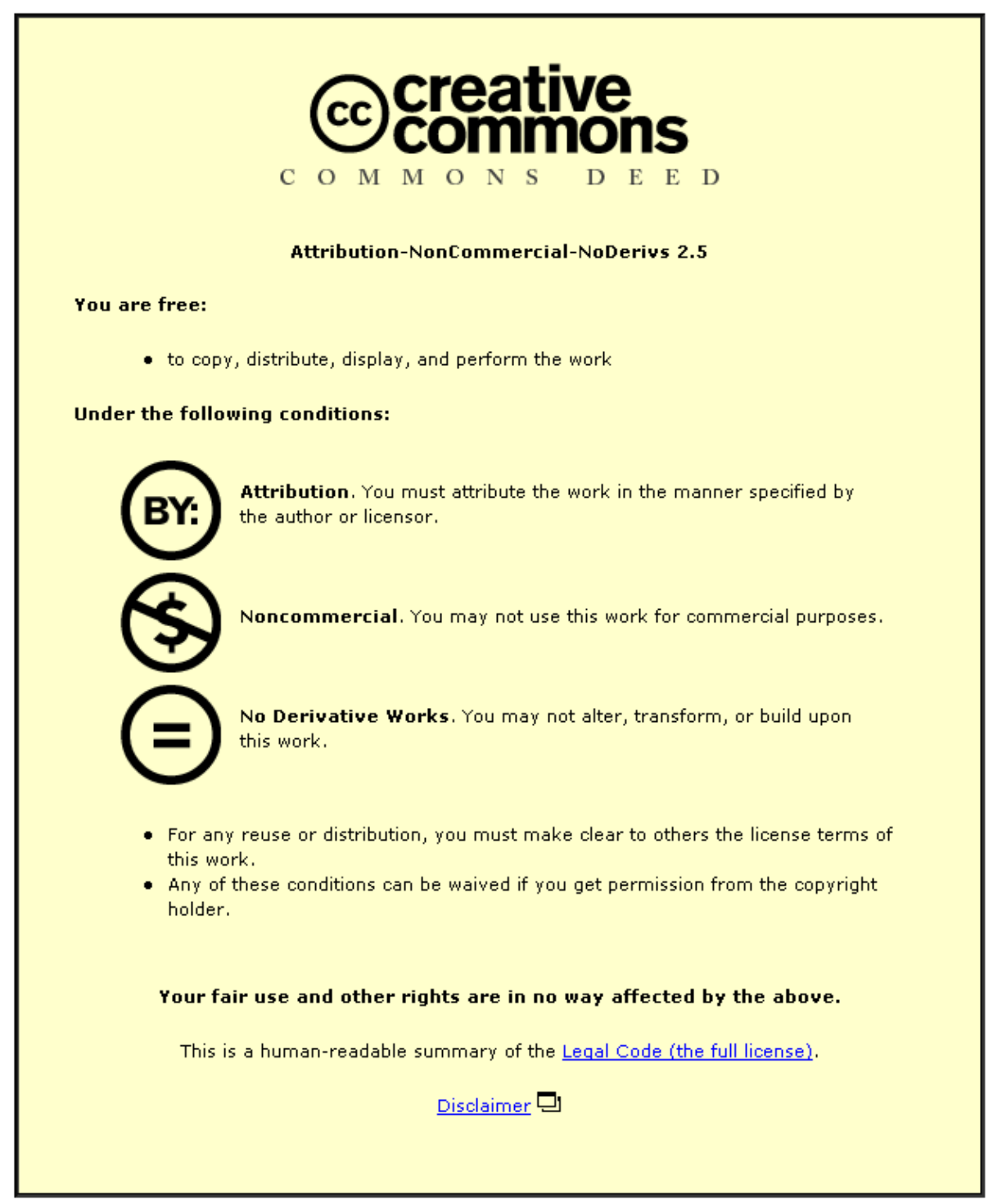

For the full text of this licence, please go to: http://creativecommons.org/licenses/by-nc-nd/2.5/ 


\title{
Distributed Quasi-Orthogonal Space-time coding for Two-Way Wireless Relay Networks
}

\author{
F. Abdurahman, A.Elazreg, and J.A.Chambers \\ Advanced Signal Processing Group, \\ Electronic and Electrical Engineering Department \\ Loughborough University, Leicestershire, UK,LE11 $3 T U$ \\ F.M.Abdurahman@lboro.ac.uk \\ A.Elazreg@iboro.ac.uk \\ J.A. Chamberselboro.ac.uk
}

\begin{abstract}
The contribution in this paper is to consider distributed quasi orthogonal space-time block coding (D-QO-STBC) for two-way (TW) wireless relay networks. In particular we exploit a two time slot protocol and both open-loop and closedloop D-QO-STBC with full cooperative diversity. In the openloop approach constellation rotation is exploited to improve performance, whereas two feedback terms are used in the closedloop schemes. Our end-to-end bit error rate simulations show that TW closed-loop D-QO-STBC and rotated open-loop D-QO-STBC are approximately $8 \mathrm{~dB}$ and $7.5 \mathrm{~dB}$ better than the distributed Alamouti TW approach at $10^{-4}$ bit error rate (BER), which confirms the advantage of four relay schemes in relay networks.
\end{abstract}

Index Terms-Cooperative relay networks, distributed quasiorthogonal space-time block coding, open-loop, exact phase feedback.

\section{INTRODUCTION}

Space time block coding (STBC) is a common technique applied in multi-input multi-output (MIMO) wireless communication systems, which has proved to be a very effective technique to leverage spatial diversity and provide full data rate [1][2]. Owing to cost constraints, size limitation and hardware complexity, it is usually difficult to co-locate multiple antennas at one mobile communication node. It is, moreover, hard to achieve spatially uncorrelated channels in many point-to-point MIMO systems. However, STBC can be extended to cooperative systems [3], in the form of distributed space time block coding (D-STBC). By sharing antennas of different users, the reliability of transmission and full transmitter diversity can thereby be achieved [3][4][5]. The most widely used D-STBC strategies are focused on the case of two relay nodes using the Alamouti scheme [1], which provides half data rate and full cooperative diversity equal to the number of relays. Cooperative diversity was proposed to improve the reliability and capacity of transmission [4] and [6] without using multiple antennas at the individual nodes. There are essentially three methods of cooperative diversity amplify-and-forward [4],[7], decode-and-forward (DF) [6],[4] and coded cooperation. Jafarkhani [8], Tirkkonen, Boariu and Hottinen [9] proposed STBC from quasi-orthogonal designs, where the orthogonality is relaxed to provide higher symbol transmission rate. With the quasi-orthogonal structure, the maximum likelihood (ML) decoding at the receiver can be performed by using pairwise decoding as in [8], but full diversity is not obtained. Su and Xia in [10] design QO-STBC with full diversity and fast ML decoding, they proposed that half of the symbols in a quasi-orthogonal design are from a signal constellation $\mathrm{A}$ and another half of them are selections from the rotated constellation $e^{j \Phi}$ set A. Two-way communication is another common communication method where two terminal nodes exchange their information between each other [11]. The TW channel was first considered by Shannon [12], who derived inner and outer bounds on the capacity region. Recently, considering the TW channel over relay networks (TWRC) has increased the interest from both academic and industrial communities [13]-[15] due to its potential application to enable range-rate enhancements of future cellular systems. By considering TWRC as a component of a general wireless network, TWRC could also lead to network resources saving in multi-hop networks such as sensor networks and ad hoc networks. In this paper, based on [10] we propose distributed rotated open-loop QO-STBC for two-way wireless relay networks so that overall unity rate is achieved with full cooperative diversity in each hop. We also propose distributed closed-loop QO-STBC for two-way wireless relay networks to obtain full cooperative diversity with array gain. The paper is organized as follows, Section II describes the system model of the TW relay wireless network. In Section III distributed open-loop quasi-orthogonal protocol using two time slots is introduced. In Section IV distributed closed-loop quasi-orthogonal protocols using two time slots are presented. Simulation results are shown in Section V. The final section summarizes this paper. In this paper, $[.]^{T}$, $[.]^{*},||,. \Re\{$.$\} and \{.\}^{H}$ denote transpose,conjugate, absolute value, real part of a complex number and Hermitian (complex conjugate transpose) operations, respectively. $C N\left(0, \sigma^{2}\right)$ represents a Gaussian distributed complex random variable with zero mean and standard variance of $\sigma^{2}$ (i.e. $0.5 \sigma^{2}$ per dimension). 


\section{SySTEM MODEL}

Consider a wireless network with four relay nodes $R_{i}$, $i=1$ to 4 , and two terminal nodes $T_{m}, m=1,2$ as shown in Fig.1. Every node has a single antenna, which can not be used for transmit and receive simultaneously(half duplex). We assume that the uplink channel gain from $T_{m}$ to $R_{i}$ is identical to the downlink channel gain from $R_{i}$ to $T_{m}$. Denote the channel from $T_{1}$ to $R_{i}$ as $f_{i}$, and the channel from $T_{2}$ to $R_{i}$ as $g_{i}$. We assume that the channels and the noise are Rayleigh flat fading, $f_{i}$ and $g_{i}$ are independent and identically distributed (i.i.d) with zero mean and unit variance. There is no direct link between the $T_{1}$ and $T_{2}$ as we assume the distance and/or shadowing is such that reliable transmission is impossible. We also assume that the channel information is unknown at the relays but perfect channel information, $f_{i}$ and $g_{i}$, is available at both terminals [16]. Assuming that terminal $T_{m}$ wants to send the signal

$\mathbf{S}_{m}=\left[s_{m, 1}, \cdots, s_{m}, k\right]^{T}$ to the other terminal, where $s_{m, t} \in A_{m}, m=1,2, t=1, \cdots, k, A_{m}$ is a finite constellation with average power 1 ,and $\mathrm{k}$ is the length of each time slot. $E\left\{\mathbf{S}_{m}^{H} \mathbf{S}_{m}\right\}=k$ [11]. We assume relays use a distributed space-time protocol. When all relay nodes receive signals from both terminals such as the first time slot, the received signal can be expressed as

$$
\mathbf{r}_{i}=\sqrt{P_{T_{1}} T} f_{i} \mathbf{S}_{1}+\sqrt{P_{T_{2}} T} g_{i} \mathbf{S}_{2}+\mathbf{n}_{r, i} \quad \text { for } i=1 \ldots N
$$

where $\mathbf{S}_{m}$ is the transmitted signal by $T_{m}, \mathbf{n}_{r, i} \sim C N(0,1)$, is the additive white Gaussian noise at the $i$ th relay node and $P_{T m}$ is the average power of terminal we assume that the total power is $\mathrm{P}, P_{1}=P_{2}=P_{R}=\frac{P}{3}$ where $P_{R}$ is the power at the relays.. We assume that all the noise terms in this paper are additive zero-mean white circular complex Gaussian variables with unity variance (i.e., $\sigma^{2}=1$.

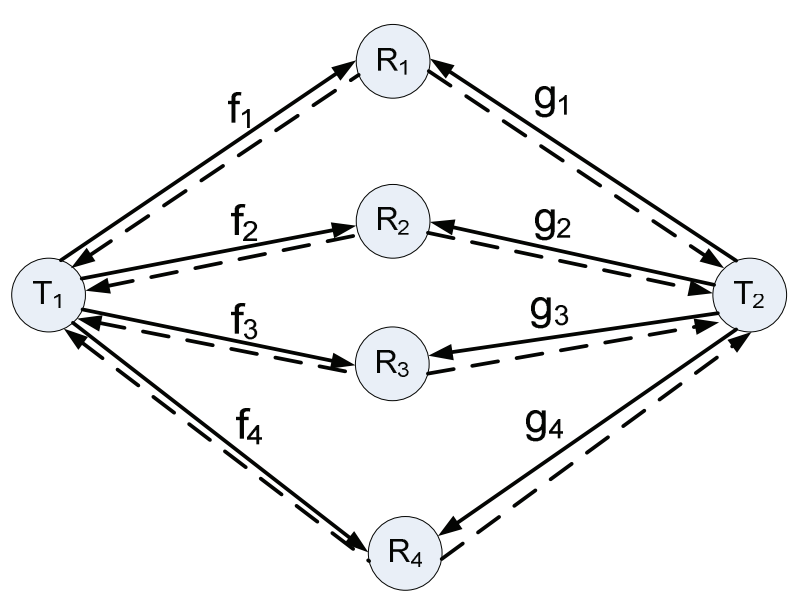

Fig. 1. Basic structure of two-way relay wireless network for uplink channel from the terminals to the relays (solid) and downlink channel from the relays to the terminals (dashed) for open-loop D-QO-STBC..

The relay node amplifies the received signal and is designed to be a linear function of its received signal and its conjugate and broadcasts it to the two terminals and can be expressed as.

$$
\mathbf{t}_{i}=\beta\left(A_{i} \mathbf{r}_{i}+B_{i} \mathbf{r}_{i}^{*}\right)
$$

where the matrices $A_{i}$ and $B_{i}$ are two $\mathrm{T}$ x $\mathrm{T}$ matrices and are used to perform the D-QO-STBC at the $i$-th relay [16] and $\beta$ is the amplification factor to maintain an average transmit power $P_{R}$ at the relay nodes which can be represented as $\beta=\sqrt{\frac{2 P_{R}}{N\left(2 P_{T_{1}}+2 P_{T_{2}}+1\right)}}$ [11]. At the second time slot both terminals will receive signals from the $i$ th relay, the received signal can be expressed as

$$
\begin{aligned}
& \mathbf{x}_{T_{1}}=\sum_{i=1}^{N} f_{i} \mathbf{t}_{i}+\mathbf{w}_{T_{1}} \\
& \mathbf{x}_{T_{2}}=\sum_{i=1}^{N} g_{i} \mathbf{t}_{i}+\mathbf{w}_{T_{2}}
\end{aligned}
$$

where $\mathbf{t}_{i}$ is the transmitted signal by the $i$ th relay node, and $\mathbf{w}_{T_{m}} \sim C N(0,1)$ is the additive white Gaussian noise at $T_{m}$. From equations (3) and (4), the received signals at the two terminals can be re-written as follows

$$
\begin{aligned}
& \mathbf{x}_{T_{1}}=\beta\left(\sqrt{2 P_{T_{2}}} S_{T_{2}} \mathbf{H}_{T_{2}}+\sqrt{2 P_{T_{1}}} S_{T_{1}} \mathbf{H}_{m}\right)+\mathbf{W}_{T_{1}} \\
& \mathbf{x}_{T_{2}}=\beta\left(\sqrt{2 P_{T_{1}}} S_{T_{1}} \mathbf{H}_{T_{1}}+\sqrt{2 P_{T_{2}}} S_{T_{2}} \mathbf{H}_{m}\right)+\mathbf{W}_{T_{2}}
\end{aligned}
$$

where

$$
\begin{aligned}
\mathbf{H}_{T m} & =\left[\begin{array}{lll}
\hat{f}_{1} \hat{g}_{1} & \ldots \ldots \ldots & \hat{f}_{R} \hat{g}_{R}
\end{array}\right]^{T} \\
\mathbf{H}_{m} & =\left[\begin{array}{llll}
\hat{h}_{1}^{2} & \ldots \ldots \ldots & h_{R}^{2}
\end{array}\right]^{T}
\end{aligned}
$$

and

$$
S_{T_{m}}=\left[\begin{array}{lll}
\hat{A}_{1} \hat{S}_{m}^{i} \ldots \ldots \ldots \hat{A}_{N} \hat{S}_{m}^{N}
\end{array}\right] m=1,2
$$

and

$$
h_{i}=\left\{\begin{array}{lll}
f_{i} & \text { if } & m=1 \\
g_{i} & \text { if } & m=2
\end{array}\right.
$$

and

$$
\mathbf{W}_{T_{m}}=\beta \sum_{i=1}^{R} h_{i} \hat{A}_{i} \hat{\mathbf{v}}_{i}+\mathbf{w} .
$$

The maximum-likelihood (ML) decoding is

$$
\arg \min _{\breve{S} \in A_{1}^{N}}\left\|\mathbf{x}_{T_{m}}-\beta\left(\sqrt{2 P_{T_{m}}} \check{S} H_{T m}+\sqrt{2 P_{T_{m}}} S_{m} H_{m}\right)\right\|^{2}
$$

where $\check{S}$ is the possible code matrices for QPSK.

\section{Distributed QuAsi-ORThogonal Rotated OPEN-LOOP PROTOCOLS FOR TW USING TWO-TIME SLOTS}

We consider the four relay node mode D-QO-STBC for two-way communications as shown in Fig.1, we assume that there is no channel state information (CSI) at the relays, but full CSI at the both terminals. In the first time slot, both $T_{1}$ and $T_{2}$ send their information to all the relays simultaneously. 
The QO-STBC code word which has to be generated by the relays has the following structure

$$
S_{T_{m}}^{i}=\left[\begin{array}{rrrr}
x_{1} & x_{2} & x_{3} & x_{4} \\
-x_{2}^{*} & x_{1}^{*} & -x_{4}^{*} & x_{3}^{*} \\
-x_{3}^{*} & -x_{4}^{*} & x_{1}^{*} & x_{2}^{*} \\
x_{4} & -x_{3} & -x_{2} & x_{1}
\end{array}\right]
$$

where $x_{1}, x_{2} \in \mathrm{A}$ and $x_{3}, x_{4} \in e^{j \Phi} \mathrm{A}$ for some signal constellation $\mathrm{A}$, and the rotation angle $\Phi$ is determined by the signal constellation A [10]. In the second time slot, the relays $R_{i}$ transmit the received information from both terminals scaled by $\beta$ to maintain average power $P_{R}$. The QO-STBC we propose achieves full rate and full diversity. In our model the four relay nodes are designed to use the following matrices as in [16]:

$$
\begin{gathered}
A_{1}=I_{4} A_{2}=0_{4} A_{3}=0_{4} A_{4}=\left[\begin{array}{llll}
0 & 0 & 0 & 1 \\
0 & 0 & -1 & 0 \\
0 & -1 & 0 & 0 \\
1 & 0 & 0 & 0
\end{array}\right] \\
B_{1}=0_{4} B_{2}=\left[\begin{array}{llll}
0 & -1 & 0 & 0 \\
1 & 0 & 0 & 0 \\
0 & 0 & 0 & -1 \\
0 & 0 & 1 & 0
\end{array}\right] \\
B_{3}=\left[\begin{array}{llll}
0 & 0 & -1 & 0 \\
0 & 0 & 0 & -1 \\
1 & 0 & 0 & 0 \\
0 & 1 & 0 & 0
\end{array}\right] B_{4}=0_{4}
\end{gathered}
$$

where the matrices $A_{k}$ and $B_{k}$ are two $4 \times 4$ matrices that depend on the D-STBC. In this paper, the special cases when either $A_{k}=0$ and $B_{k}$ is unitary or $B_{k}=0$ and $A_{k}$ is unitary are considered as in [16]. Under this special case, the $i$-th column of the code matrix can contain either the information symbols or their conjugates only, as in (11) and the following variable can be defined as follow

$$
\left.\begin{array}{l}
\hat{A_{k}}=A_{k}, \quad \hat{f}_{k}=f_{k}, \quad \hat{g}_{k}=g_{k} \\
\hat{h}_{k}=h_{k}, \quad \hat{v}_{k}=v_{k}, \quad \mathbf{s}_{T_{j}}^{(i)}=\mathbf{s}_{T_{j}}
\end{array}\right\} \quad \text { if } B_{k}=0 \text { (14) }
$$

and

$$
\left.\begin{array}{l}
\hat{A_{k}}=B_{k}, \quad \hat{f}_{k}=f_{k}^{*}, \quad \hat{g}_{k}=g_{k}^{*} \\
\hat{h}_{k}=h_{k}^{*}, \quad \hat{v}_{k}=v_{k}^{*}, \quad \mathbf{s}_{T_{j}}^{(i)}=\mathbf{s}_{T_{j}}^{*}
\end{array}\right\} \quad \text { if } A_{k}=0
$$

Therefore the received signal can be represented as shown in (5) and (6), and the channel matrix in (5) and (6) can be written as follows

$$
\begin{array}{r}
\mathbf{H}_{T m}=\left[\begin{array}{llll}
\hat{f}_{1} \hat{g}_{1} & \hat{f}_{2} \hat{g}_{2} & \hat{f}_{3} \hat{g}_{3} & \hat{f}_{4} \hat{g}_{4}
\end{array}\right]^{T} \\
\mathbf{H}_{m}=h_{k}\left[\begin{array}{llll}
\hat{h}_{1} & \hat{h}_{2} & \hat{h}_{3} & \hat{h}_{4}
\end{array}\right]^{T}
\end{array}
$$

and the noise vector can be written as in (11). Pairwise decoding can be used as described in [8].

\section{Distributed Quasi-Orthogonal Closed-Loop Protocols For TW Using Two-Time Slots}

In order to achieve full diversity, the data signals transmitted from the third and fourth relays, which are denoted as $t_{3}$ and $t_{4}$ are multiplied by $U_{1}$ and $U_{2}$, as shown in Fig. 2, before they are transmited to both terminals, while the other two relay nodes are kept unchanged. In our model we adopt a feedback scheme as in [17] to determine the value of $U_{1}$ and $U_{2}$ as follows $U_{1}=e^{j \theta_{1}}$ and $U_{2}=e^{j \theta_{2}}$, where $\theta_{1}$ and $\theta_{2}$ are phase rotation angles.

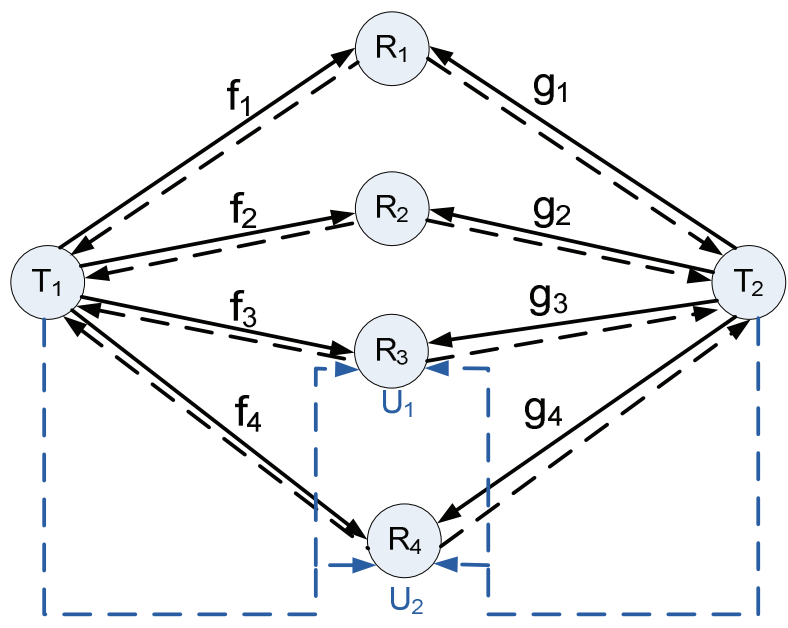

Fig. 2. Basic structure of two-way relay wireless network for uplink channel from the terminals to the relays (solid) and downlink channel from the relays to the terminals (dashed) for closed-loop D-QO-STBC , and feedback (light-dashed).

Therefore, the data signals transmitted from the relays to $T_{1}$ and $T_{2}$ as in (2) can be written as:

$$
\begin{aligned}
\mathbf{t}_{i} & =\beta\left(\sum_{k=1,2} \sqrt{P_{T_{1}}} \hat{f}_{k} \hat{A}_{k} \mathbf{S}_{T_{1}}^{(i)}+\sum_{k=3,4} \sqrt{P_{T_{1}}} U_{k-2} \hat{f}_{k} \hat{A}_{k} \mathbf{S}_{T_{1}}^{(i)}\right) \\
& +\beta\left(\sum_{k=1,2} \sqrt{P_{T_{2}}} \hat{g}_{k} \hat{A}_{k} \mathbf{S}_{T_{2}}^{(i)}+\sum_{k=3,4} \sqrt{P_{T_{2}}} U_{k-2} \hat{g}_{k} \hat{A}_{k} \mathbf{S}_{T_{2}}^{(i)}\right) \\
& +\beta\left(\sum_{k=1,2} \hat{A}_{k} \hat{\mathbf{v}}_{k}+\sum_{k=2,4} U_{k-2} \hat{A}_{k} \hat{\mathbf{v}}_{k}\right)
\end{aligned}
$$

Therefore, the received signals at both terminals can be represented as in (5) and (6), where the channel vectors in (5) and (6) can be written as:

$$
\begin{array}{r}
\mathbf{H}_{T m}=\left[\begin{array}{llll}
\hat{f}_{1} \hat{g}_{1} & \hat{f}_{2} \hat{g}_{2} & U_{1} \hat{f}_{3} \hat{g}_{3} & U_{2} \hat{f}_{4} \hat{g}_{4}
\end{array}\right]^{T} \\
\mathbf{H}_{m}=h_{k}\left[\begin{array}{llll}
\hat{h}_{1} & \hat{h}_{2} & U_{1} \hat{h}_{3} & U_{2} \hat{h}_{4}
\end{array}\right]^{T}
\end{array}
$$

where $\hat{f}_{k}$ and $\hat{g}_{k}$ can be obtained as in (14) and (15), and $h_{k}$, $k=1,2,3$ and 4 can be obtained as in (10) and the noise vectors in (5) and (6) can be written as:

$$
\mathbf{W}_{T_{m}}=\beta\left(\sum_{k=1,2} h_{m} \hat{A}_{k} \hat{v}_{k}+\sum_{k=3,4} U_{k-2} h_{m} \hat{A}_{k} \hat{v}_{k}\right)+\mathbf{w} \text {. }
$$

The decoding is as represented in (12). 


\section{Simulation Results}

In this section, we show simulated performance of distributed closed-loop D-QO-STBC and open-loop D-QOSTBC. The error measure is the end-to-end bit error rate (BER). The information symbols are modulated as QPSK. We fix the total power consumed in the whole network as $\mathrm{P}$. In Figure 3, the horizontal axis indicates the total power used in the whole network and the $\mathrm{Y}$ axis indicates BER. We assume that the total power is $\mathrm{P}, P_{T_{1}}=P_{T_{2}}=P_{R}=\frac{P}{3}$ where $P_{R}$ is the total power at the relays, and $P_{R_{i}}=\frac{P_{R}}{4}, \mathrm{i}=1, . ., 4$.

In Fig. 3, we show the BERs with QPSK symbols. Twoway closed-loop D-QO-STBC is approximately $.5 \mathrm{~dB}$ better than the rotated open-loop D-QO-STBC and about $3 \mathrm{~dB}$ and $8 \mathrm{~dB}$ better than the open-loop D-QO-STBC and the Alamouti two-way at $10^{-4} B E R$.

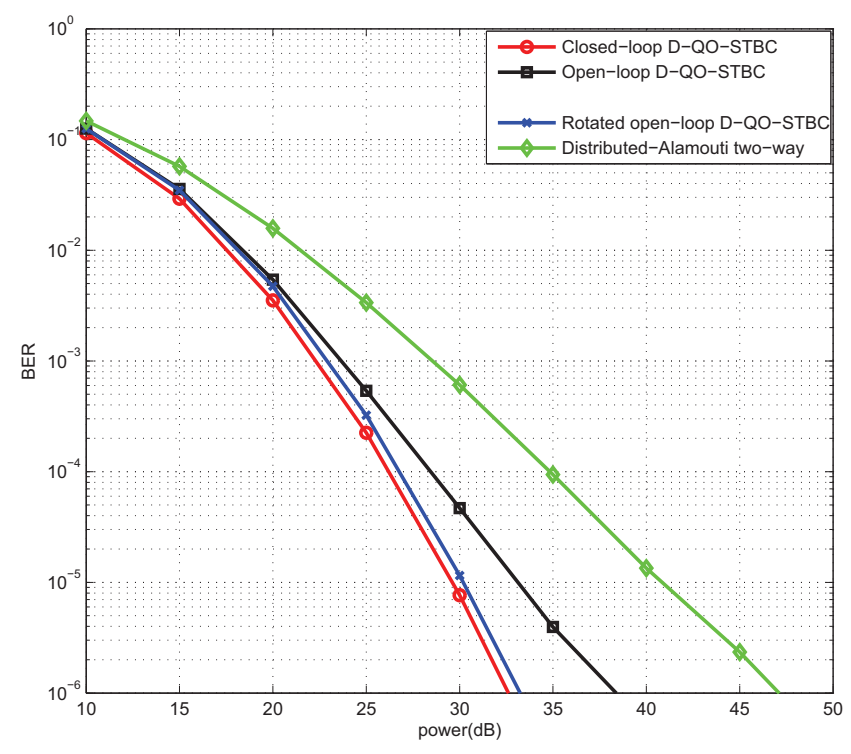

Fig. 3. Performance comparison of two-time slot protocols with Alamouti two-way and different two-way QO-STBC using QPSK.

\section{CONCLUSION}

Distributed closed-loop D-QO-STBC and open-loop D-QOSTBC transmission methods for TW wireless relay networks with four relay nodes were investigated assuming perfect synchronization. A good performance with full date rate and full diversity is achieved by using closed-loop D-QO-STBC rather than open-loop D-QO-STBC. Moreover, it has been shown that the closed-loop D-QO-STBC is .5 $\mathrm{dB}$ better than the rotated open-loop D-QO-STBC and approximately $3 \mathrm{~dB}$ and $8 \mathrm{~dB}$ better than the open-loop D-QO-STBC and the distributed Alamouti two-way. In addition to the superiority in BERs, the two-way rotated open-loop D-QO-STBC and openloop D-QO-STBC have a disadvantage in decoding complexity since the information symbols can be decoded pairwise at the receiver, while symbol wise decoding can be used in closedloop D-QO-STBC. Future work will consider the mitigation of asynchronism in the network.

\section{REFERENCES}

[1] A.M. Alamouti, "A simple transmit diversity techniques for wireless communications," IEEE J, SAC, vol.16, no.8, pp.1451-1458, 1998.

[2] V. Tarokh, H. Jafarkhani, and A. Calderbank, "Space time block codes from orthogonal design," IEEE Trans Inform Theory, vol. 45, no. 5, pp.1456-1467, 1999.

[3] J. Laneman, and G. Wornell, "Distributed SpaceTime-Coded Protocols for Exploiting Cooperative Diversity in Wireless Networks, “ IEEE Trans Inform Theory, vol.49, no. 10, pp. 2415-2425, 2003.

[4] A. Sendonaris, E. Erkip, and B. Aazhang, "User cooperation diversity part I: System description,“ IEEE Trans.on Commun., vol. 51, no. 11, pp. 1927-1938, 2003.

[5] A. Nosratinia, T.E Hunter,and A. Hedayat, "Cooperative communication in wireless networks, “ IEEE Communications Magazine, vol. 42, no. 10, pp. 74-80, 2004.

[6] K. Azarian, H. El Gamal, and P. Schniter, "On the achievable diversitymultiplexing tradeoff in half-duplex cooperative channels, " IEEE Trans. Inf. Theory, vol. 51, no. 12, pp. 4152-4172, 2005.

[7] A. F. Dana and B. Hassibi, "On the power-efficiency of sensory and ad hoc wireless networks," IEEE Trans. Inf. Theory, vol. 52, no. 7, pp.2890-2914, 2006.

[8] H. Jafarkhani, "A quasi-orthogonal space-time block codes," IEEE Trans. Commun., vol. 49, no. 1, pp. 14, 2001.

[9] O. Tirkkonen, A. Boariu, and A. Hottinen, "Minimal nonorthogonality rate 1 space-time block code for $3+$ Tx antennas," in Proc. IEEE 6th Int.Symp. Spread-Spectrum Techniques and Applications (ISSSTA 2000), 2000, pp. 429-432.

[10] W. Su and X. Xia, "Signal constellations for quasi-orthogonal spacetime block codes with full diversity," IEEE Trans. Inf. Theory, vol. 50,no. 10, pp. 2331-2347, 2004.

[11] T. Cui, F. Gao, T. Ho and A. Nallanathan "Distributed space-time coding for two-way wireless relay networks" IEEE Trans. on. Signal processing, vol. 57,no. 2,pp.658-671,2009.

[12] C. E. Shannon, "Two-way communication channels," in Proc. 4th Berkeley Symp. Math. Stat. Prob., 1961, pp. 611644

[13] B. Rankov and A. Wittneben, "Spectral efficient signaling for halfduplex relay channels," in Proc. Asilomar Conf. Signals, Systems, Computers, 2005, pp. 1066-1071.

[14] B. Rankov and A.Wittneben, "Achievable rate regions for the two-way relay channel," in Proc. IEEE ISIT, 2006, pp. 1668-1672.

[15] C. Hausl and J. Hagenauer, "Iterative network and channel decoding for the two-way relay channel," in Proc. IEEE ICC, 2006, pp.15681573.

[16] Y. Jing and H. Jafarkhani, "Using orthogonal and quasiorthogonal designs in wireless relay networks, “ IEEE Trans. on Information Theory,vol. 53, pp. 41064118, 2007.

[17] C. Toker, S. Lambotharan, and J. Chambers, "Closed-Loop quasiorthogonal STBCs and their performance in multipath fading environments and when combined with Turbo codes," IEEE Trans. on Wireless Commun., vol. 3, no. 6, pp.1890-1896,2004. 